\title{
Imaging Findings of Pulmonary Nocardiosis Mimicking Bronchiectasis
}

\author{
Lianmin Bao1, Heping Lin1, Ling Dong1, Qing Zhang2 and Zongxiao Shangguan1
}

\begin{abstract}
Nocardia species usually cause opportunistic infections, and the frequency of these infections is increasing owing to the growing population of immunocompromised hosts. However, Nocardia may sometimes causes an infectious disease in immunocompetent hosts. Herein, we report two cases of pulmonary nocardiasis in immunocompetent individuals, whose chest computed tomography (CT) findings mimicked bronchiectasis. Samples of bronchalveolar lavage (BAL) fluid obtained by bronchoscopy showed filamentous, branching, gram-positive rods, acid-fast filamentous branching rods, and a colony of suspected Nocardia was cultured. Based on 16sRNA and hsp65 gene sequence analysis, case 1 was identified as $\mathrm{N}$. cyriacigeorgica, but case 2 was not matched. The patients responded well to treatment with the combination of sulfamethoxazole and linezolid.
\end{abstract}

Key Words: Pulmonary nocardiosis, Immunocompetent, Bronchiectasis.

\section{INTRODUCTION}

Pulmonary nocardiosis is a clinically infrequent disease caused by organsous of Nocardia species. Clinical and radiological manifestations of pulmonary nocardiosis are nonspecific and definite microbiological data are lacking for most Nocardia species, leading to its misdiagnosis or missed diagnosis. Therefore, this disease may be underestimated. Nocardiosis typically occurs in the setting of immunocompromised conditions, but it may also affect immunocompetent individuals with structural lung disease, such as chronic obstructive pulmonary disease (COPD) and bronchiectasis. ${ }^{1}$ In recent years, the number of Nocardia infections in patients with bronchiectasis is increasing. ${ }^{2}$ However, whether pulmonary Nocardia infection masquerades as bronchiectasis has been little reported. Here, we report two cases of pulmonary nocardiosis whose imaging findings mimicked bronchiectasis, along with a review of the relevant literature, in order to improve the understanding of pulmonary nocardiosis.

\section{CASE REPORT}

Case 1: A 60-year man was referred from another institution with a 3-year history of productive cough, and 5-day history of aggravation in April 2014. Three years ago, the patient developed moderate symptoms of paroxysmal cough and sputum. The sputum was hard to

Department of Respiratory Medicinel / Clinical Laboratory ${ }^{2}$, The Third Affiliated Hospital of Wenzhou Medical University, Wenzhou, Zhejiang, China

Correspondence: Dr. Zongxiao Shangguan, Department of

Respiratory Medicine, The Third Affiliated Hospital of

Wenzhou Medical University, Wenzhou, Zhejiang, China

E-mail:309872535@qq.com

Received: June 07, 2018; Accepted: September 18, 2018 cough up. In the community hospital, he was diagnosed as pneumonia. By using antibiotics and other symptomatic treatments (details unknown), his symptoms improved. However, the symptoms recurred whenever drugs were stopped. Five days ago, the patient developed cough again with bloody sputum. Then, he presented to our hospital for further investigations. He had no history of repeated cough, blood-streaked sputum or hemoptysis in his 56-year life. He denied history of tuberculosis, and denied alcohol and tobacco intake. On physcial examination, his temperature was $36.9^{\circ} \mathrm{C}$, heart rate of $94 /$ minute, blood pressure of $156 / 78 \mathrm{mmHg}$, and respiratory rate of $20 /$ minute. Trachea was in midline. Thoracic expansion was symmetrical and normal, and a few moist rales were audible over the right lung base. Auxiliary investigations showed white blood cells (WBC) count of $4.3 \times 10^{9} / \mathrm{L}$, neutrophil percentage $57.1 \%$, hemoglobin $119 \mathrm{~g} / \mathrm{L}$, platelets $223 \times 10^{9} / \mathrm{L}$, C-reactive protein (CRP) $1.3 \mathrm{mg} / \mathrm{L}$, erythrocyte sedimentation rate (ESR) $10 \mathrm{~mm} / \mathrm{h}$, and T-SPOT, negative. Sputum failed to reveal any acid-fast bacilli for three times. T-lymphocyte subsets, immunoglobulin classes and autoantibody screen were unremarkable. Human immunodeficiency virus (HIV) was negative. Pulmonary function test was unremarkable. Chest computed tomography (CT) scan showed "bronchiectasis of middle-lobe of right lung and lingular bronchus of left lung" (Figure 1a). Two days later, he was examined by bronchalveolar lavage (BAL) of middle lobe of right lung. Cell analysis of BAL fluid showed neutrophils $90 \%$, lymphocytes $7 \%$, epithelial cells $1 \%$, macrophages $2 \%$, and eosinophils $1 \%$, indicating neutrophils predominance. BAL fluid smear was weakly positive by acid-fast stain (Figure 1b), while acid-fast stain was negative (data not shown); and bronchoscopic aspirate sample also revealed weakly acid-fast staining bacteria, where showed sulfur granule (Figure 1c). Moreover, both BAL fluid and sputum culture 


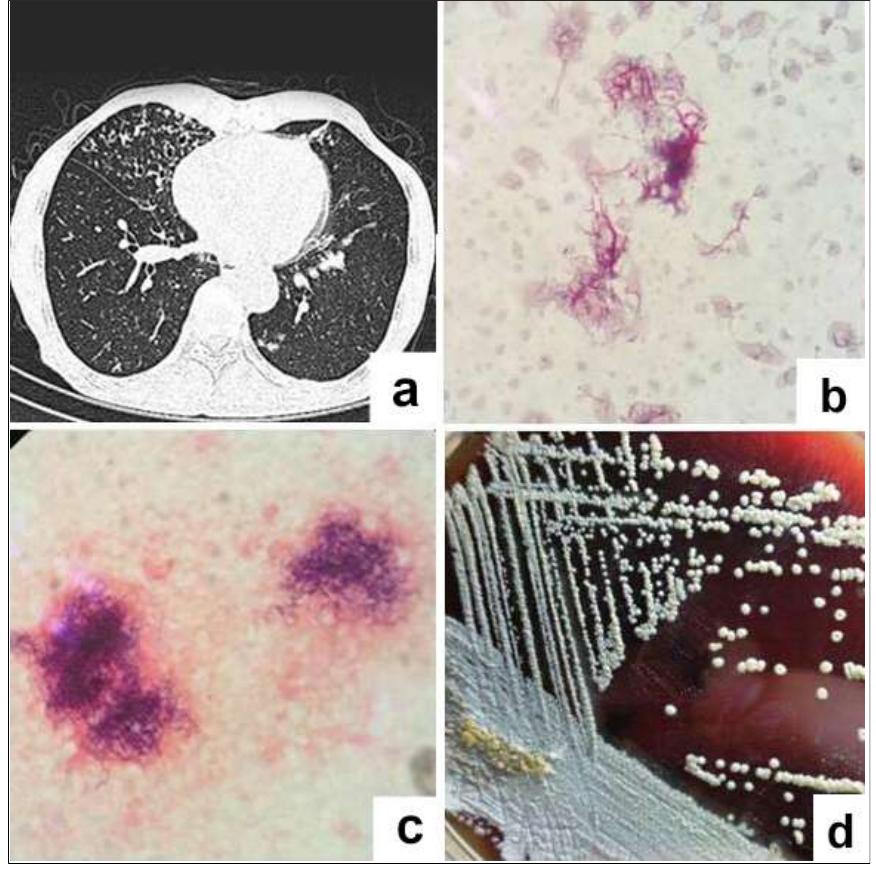

Figure 1: (a) Chest CT scan of case 1 showing bronchiectasis of middle-lobe of right lung and lingular bronchus of left lung; (b) Weakly acid-fast stain of Nocardia in BAL fluid demonstrating delicate beaded branching filaments; (c) Weakly acid-fast stain in bronchoscopic aspirate sample demonstrating sulfur granules; (d) Nocardia growth in BAL fluid culture.

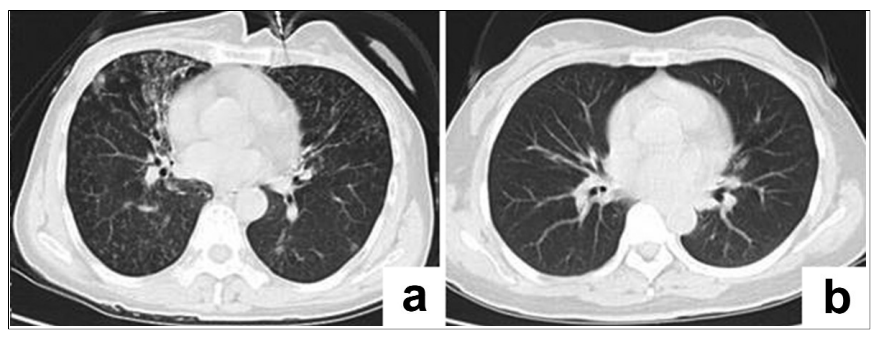

Figure 2: (a) Chest CT scan of case 2 showing bronchiectasis of middle-lobe of right lung and lingular bronchus of left lung; (b) Chest CT scan of case 2 in 2011, showing non-dilated airways.

revealed Nocardia growth (Figure 1d). Using 16sRNA and hsp65 gene sequence analysis, the homology of N. cyriacigeorgica was 100\% (FO082843.1) and 99\% (EF127506.1) in PubMed Blast. The patient was diagnosed with pulmonary nocardiosis, sulfamethoxazole $(0.96 \mathrm{~g}$, 3times/day) and linezolid (0.6g, 2times/day) were used for anti-infection therapy. After 8 days of treatment, his condition became improved, cough and sputum were reduced, hemoptysis, fever and other symptoms were subsided. He was discharged with drugs (sulfamethoxazole + linezolid) for 8 weeks, following-up for 6 months without any symptoms relapsed.

Case 2: A 53-year female patient presented with a 5 -year history of repeated cough and hemoptysis, and relapse for 5 days in February, 2017. Five years ago, this woman developed cough with slight yellow-green sputum, sometimes with bloody sputum. She was diagnosed as bronchiectasis in the local clinic. After symptomatic treatment (drugs unknown), her symptoms were improved. However, these symptoms were recurrent, and she received anti-infective and hemostatic treatment in the outpatient department of our hospital for several times. Five days ago, the recurrence of bloody sputum made her receive inpatient therapies. The chest CT scan showed "bronchiectasis of middlelobe of right lung and lingular bronchus of left lung" (Figure 2a). We reviewed her chest CT examined in 2011 in our hospital, which showed that airways were not dilated (Figure 2b). She denied history of tuberculosis, alcohol and tobacco intake. On physical examination, her temperature was $36.5^{\circ} \mathrm{C}$, heart rate of 77 beats/minute, blood pressure of $125 / 80 \mathrm{mmHg}$, and respiration rate of 19 breaths/minute. Thoracic expansion was symmetrical and normal, and a few moist rales were audible over right lung. Auxiliary investigations showed white cell count of $7.1 \times 109 / \mathrm{L}$, with neutrophils $64.3 \%$, lymphocytes $25.7 \%$, hemoglobin $120 \mathrm{~g} / \mathrm{L}$, platelets $245 \times 10^{9} / \mathrm{L}$, CRP $5.7 \mathrm{mg} / \mathrm{L}$, ESR $15 \mathrm{~mm} / \mathrm{h}$, and total $\mathrm{lgE}$ level of $206.2 \mathrm{IU} / \mathrm{ml}$. Sputum smear test was negative for acid-fast bacilli 3 times. However, sputum showed grampositive bacilli, which were radially arranged, and surrounded by large number of white blood cells. TSPOT was negative, and was HIV serology. Three days later, she was examined by bronchoalocive lavage (BAL) of middle lobe of right lung. Cell analysis of BAL fluid showed neutrophils $94 \%$, lymphocytes $2 \%$, epithelial cells $1 \%$, and macrophages $3 \%$. BAL fluid smear revealed weakly acid-fast positive bacteria. BAL fluid culture showed Nocardia growth. We failed to identify subtype by sequence analysis. The patient was diagnosed with pulmonary nocardiosis. Sulfamethoxazole $(0.96 \mathrm{~g}, 3$ times/day) and linezolid $(0.6 \mathrm{~g}$, 2 times/day) were used. After 15 days of treatment, her condition improved. She was discharged with drugs (sulfamethoxazole + linezolid) for 8 weeks. Now, she is still under follow-up visit, and she is symptom-free.

\section{DISCUSSION}

The genus Nocardia belongs to the family Nocardiaceae, which compresses of aerobic, gram-positive, mycolic acid-containing actinomycetes. ${ }^{3}$ The classic infectious route of Nocardia is via the respiratory tract (by inhalation). Skin, gastrointestinal invasion, and personto-person transmission is rare. The major pathogenic strains include N.asteroides, N.brasiliensis, N.otitidiscaviarum, N.farcinica, N.nova, N.cyriacigeorgica and N.mexicana. Pulmonary nocardiosis is usually produced by N.asteroides $(85 \%),{ }^{4}$ whereas N.farcinica is more frequently involved in brain and skin infections. 5 Cell mediated immunity is important in preventing dissemination of infection and killing the bacteria in the early stage of tissue invasion, 6 making nocardiosis an opportunistic infection. However, up to one-third of patients may be immunocompetent, especially 
those with underling structural lung diseases such as chronic obstructive pulmonary disease (COPD) and bronchiectasis. ${ }^{1}$ These preexisting structural abnormalities of lung cause respiratory immune system dysfunction and facilitate lower respiratory tract infection and airway colonisation. 5

Nocardiosis is a severe infection that commonly presents as subacute or chronic suppurative disease. The lungs are the most common site of nocardiosis. In an immunocompromised host, nocardiosis can progress to tissue invasion and dissemination; however, immunocompetent individuals rarely develop systemic infection, resulting in poor prognosis, especially if central nervous system is involved. ${ }^{2}$ In such cases, the mortality rate can reach $40 \% \sim 87 \% .^{7}$ Hence, cerebrospinal fluid (CSF) examination or brain CT/MRI should be performed in cases of pulmonary nocardiosis. In the present report, two patients presented with productive cough and bloody sputum. No other symptoms were observed. Brain CT scans were unremarkable (data not shown), so we did not perform CSF examination.

The signs, symptoms, and radiologic characteristics may suggest the diagnosis of nocardiosis, but are not pathognomonic. The key to diagnosing pulmonary nocardiosis is based on isolation of Nocardia species in sputum, BAL fluid or other respiratory secretions. ${ }^{5}$ Sputum cultures are rarely false-positive, however, the positive rate is only $2 / 5$ to $1 / 2$ of patients. Use of invasive diagnostic techniques can significantly improve the detection rate of Nocardia, such as BAL, fiberoptic bronchoscopy biopsy, and percutaneous lung biopsy. BAL fluid cultures showed Nocardia growth in both patients in this report. Clinical laboratory should further use molecular methods for identification of Nocardia genus at the species level, because the biological characteristics may be significantly different, especially in drug resistance and pathogenicity. In the present paper, both cases were identified by 16 sRNA and hsp65 sequence analysis, which is the gold standard. The strain of case 1 was matched with N.cyriacigeorgica, but case 2 patient was not specifically matched.

Trimethoprim/sulfamethoxazole (TMP-SMX) is the firstline treatment for pulmonary nocardiosis. However, drug resistance has gradually increased. Uhde et al. observed TMP-SMX resistance in $42 \%$ cases. ${ }^{8}$ Evidence from experimental studies using antimicrobial combinations has demonstrated synergy in vitro. Pulmonary nocardiosis treatment is treated by combining sulfonamides with carbapenems or linezolid. In this report, both patients were treated successfully with sulfamethoxazole combined with linezolid.

The radiological appearances of pulmonary nocardiosis are nonspecific. Chest CT imaging commonly shows nodules, cavitated masses, consolidations and interstitial patterns. ${ }^{9}$ Bronchiectasis is a risk factor for Nocardia infections. It is possible that bronchiectasis weakens airway cilia swing, impairs airway epithelial cells, and leads to bacterial colonisation. So far, many cases of bronchiectasis-infected nocardiosis have been reported, but these studies pay more attention to clinical manifestations and chest radiological images instead of Nocardia infections, leading to misdiagnosis or missed diagnosis. The two cases in this paper were both middleaged patients. Case 1 had no prior history of repeated productive cough, hemoptysis and other symptoms of bronchiectasis. Case 2 never had signs and symptoms of bronchiectasis before. So, we speculate that bronchiectasis may be one of the imaging manifestations of pulmonary nocardiosis. Although it is unclear whether Nocardia infection can contribute to the development or progression of bronchiectasis.

The prognosis of pulmonary nocardiosis is related to immune status, underlying diseases, systemic dissemination and treatment. In the present cases, we investigated the immune status, and both patients had normal level of immunocompetence for steroid independence and they were HIV negative. Finally, both received combined treatment with favourable prognosis.

\section{REFERENCES}

1. Martinez Tomas R, Menendez Villanueva R, Reyes Calzada S, Santos Durantez M, Valles Tarazona JM, Modesto Alapont M, et al. Pulmonary nocardiosis: risk factors and outcomes. Respirology 2007; 12:394-400.

2. Woodworth MH, Saullo JL, Lantos PM, Cox GM, Stout JE. Increasing nocardia incidence associated with bronchiectasis at a tertiary care center. Ann Am Thorac Soc 2017; 14:347-54.

3. You Y, Chen W, Zhong B, Song Z, Yang X. Disseminated nocardiosis caused by Nocardia elegans: $A$ case report and review of the literature. Infection 2018; 46:705-10

4. Beaman BL, Burnside J, Edwards B, Causey W. Nocardial infections in the United States, 1972-1974. J Infect Dis. 1976; 134:286-9.

5. Fujita T, Ikari J, Watanabe A, Tatsumi K. Clinical characteristics of pulmonary nocardiosis in immunocompetent patients. $J$ Infect Chemother 2016; 22:738-43.

6. Menendez R, Cordero PJ, Santos M, Gobernado M, Marco V. Pulmonary infection with Nocardia species: A report of 10 cases and review. Eur Respir J 1997; 10:1542-6.

7. Kim SY, Lee KL, Lee DM, Jeong JH, Moon SM, Seo YH, et al. Erratum: Nocardia brain abscess in an immunocompetent patient. Infect Chemother 2015; 47:304

8. Uhde KB, Pathak S, McCullum I, Jannat-Khah DP, Shadomy SV, Dykewicz CA, et al. Antimicrobial-resistant nocardia isolates, United States, 1995-2004. Clin Infect Dis 2010; 51: 1445-8.

9. Blackmon KN, Ravenel JG, Gomez JM, Ciolino J, Wray DW. Pulmonary nocardiosis: Computed tomography features at diagnosis. J Thorac Imaging 2011; 26:224-9.

$$
\text { ........ }
$$

